\title{
Materials science approaches in the development of broad-spectrum antiviral therapies
}

The COVID-19 pandemic has reignited efforts to develop materials science innovations aimed at stopping viral infections. One of the greatest opportunities lies in developing broad-spectrum antiviral technologies that work against many viruses, which could be the key to thwarting outbreaks in the future.

\section{Nam Joon Cho and Jeffrey S. Glenn}

$\mathrm{n}$ the twenty-first century, we have already experienced several viral outbreaks and pandemics that have challenged us to rethink how we stop virus infections from spreading. Despite our best efforts, the 2009 swine flu pandemic is estimated to have affected a greater number of people than even the 1918 Spanish flu pandemic ${ }^{1}$. We have also witnessed outbreaks of highly fatal viruses such as Ebola $^{2}$ and unexpected ones such as Zika ${ }^{3}$. Together, these experiences have taught us that no matter how prepared we are, the unexpected will always happen and we must be ready to rapidly deal with emerging and re-emerging viruses, sometimes in the absence of effective diagnostics, therapeutics and vaccines.

The ongoing coronavirus outbreak is the latest example highlighting these needs and challenges, especially as new infection cases have emerged worldwide at breakneck speed, and the World Health Organization has officially declared COVID-19 a

pandemic. Indeed, the situation has quickly spiralled out of control in recent weeks and while some countries have started to see a reduction in their number of new infections, other countries are now witnessing an exponential growth in theirs ${ }^{4}$. The situation has become more challenging as reports show that infected people without symptoms or only mild illness can spread the virus to others ${ }^{5}$. There are growing calls to stop the virus, and the public is looking for effective solutions beyond what science and medicine can provide today. In this Comment, we introduce some of the latest broad-spectrum antiviral strategies - mainly still in the lab but in some cases in clinical trials or on the cusp of approval - that might be useful to address the medical challenges associated with emerging viral pathogens like novel coronavirus (SARS-CoV-2) and to thwart them in the near future.

While the concept of broad-spectrum antivirals has long been covered in virology and the search for promising small
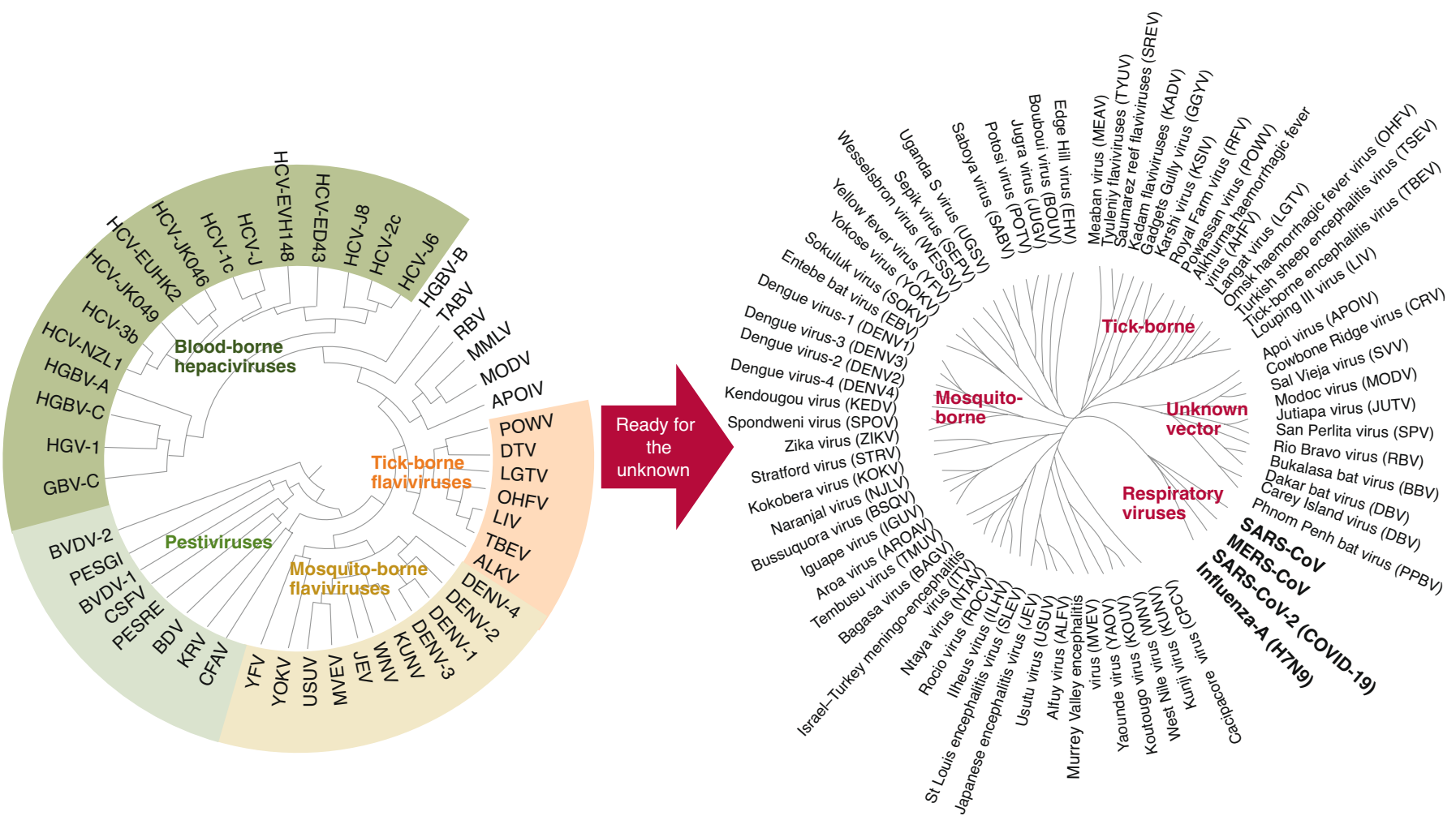

Fig. 1 | Overview of emerging viral pathogens. Pandemic preparedness planning must account for not only known viruses but also currently unknown and understudied viruses. Broad-spectrum antiviral strategies that work against a wide range of viruses have excellent potential as first-line countermeasures. 
molecules continues, we pay particular attention to recent examples from the materials science and engineering field that highlight how other classes of antiviral drug candidates, such as engineered peptides and nanoparticles, demonstrate the potential to inhibit a wide range of medically important viruses and might be used to stop viral pandemics in the future. We hope such examples can accelerate the development and implementation of next-generation antiviral strategies and inspire researchers from the materials science community to concentrate efforts on solving problems related to emerging viral pathogens. To date, there has been scant progress in translating materials science innovations into viable antiviral drug candidates and we urge closer cooperation and knowledge-sharing across the two communities that can lead to clinically meaningful outcomes. The clinical needs are there and we must work together across traditional scientific boundaries to realize solutions.

\section{COVID-19 needs and realities}

Today, as COVID-19 is spreading globally, scientists around the world are racing to develop a vaccine ${ }^{6}$. However, it will take a long time, perhaps years, to develop, test and deploy a new vaccine whose safety has been sufficiently validated, and eventual success is uncertain ${ }^{7}$. A new vaccine also raises questions about potential issues like antibody-dependent enhancement (ADE) should a vaccinated person become infected with another coronavirus ${ }^{8}$. Issues like ADE require careful attention. Likewise, there are a large number of human clinical trials taking place worldwide to test the efficacy of various experimental therapeutics for treating COVID-19 infections and caution is warranted to ensure that the best candidates are prioritized for the sake of patient safety, maximizing the chance of treatment success, and ensuring statistical significance to prove or refute the potential of a particular treatment option to guide future clinical decisions.

There has also been some progress in initiating human clinical trials of repurposed drugs, but in some cases, the repurposed drug (for example, remdesivir) had rather inferior efficacy against its initial virus target ${ }^{9}$, leaving doubts about whether such drugs will be clinically effective or merely attempts at salvaging past drug development projects $^{10,11}$. In the case of remdesivir, such prospects have been further dimmed by rather disappointing results in initial test outcomes for three COVID-19 patients who received remdesivir in the United States, as compared to other patients who did not receive the drug ${ }^{11}$. The repurposing of other pharmaceutical drugs like hydroxychloroquine has also received attention to treat patients with COVID-19 infections, although different studies have shown varying levels of clinical benefits ranging from negligible to accelerated recovery ${ }^{12,13}$.

There are also concerns about how mutations in virus structure could affect virulence and transmissibility, with at least one report suggesting that SARS-CoV-2 might have already diverged into more and less virulent strains ${ }^{14}$. As such, the strain of coronavirus we target today could look different tomorrow, potentially curtailing the impact of ongoing vaccine and drug development efforts.

\section{How do we deal with an unknown virus?}

The unexpected nature of how viral outbreaks arise and the urgent need to blunt them shares many parallels with the challenges faced in global society, for which management experts have coined the acronym VUCA, which stands for volatility, uncertainty, complexity and ambiguity ${ }^{15}$. The VUCA concept defines the landscape in which we live and work, encompassing the range of issues we deal with in daily life such as moving forward into situations without precedent, encountering rapidly evolving circumstances and facing complex global problems. Today, we are witnessing a range of VUCA examples in daily life in response to the COVID-19 pandemic, such as travel bans, mandated school and store closures, remote working arrangements, and mass quarantines. These actions, intended to stop COVID-19, are having serious consequences for global society and highlight not only how interconnected we are in today's world, but also how quickly things can fall apart when that interconnectedness comes to a screeching halt. We are living in challenging times and while the situation can appear paralysing at first glance, it should also motivate us to explore innovative ways to confront the 'unknown unknowns' by devising effective strategies not only to deal with what is happening now, but also to be prepared for what might occur (again) in the near future ${ }^{16}$ (Fig. 1).

\section{Broad-spectrum antiviral concept}

The current approach of rapid vaccine development and repurposing evaluation of approved antivirals and other drug classes is and will remain an important component of pandemic preparedness planning and should not be discounted. However, this approach by itself is not enough and we need fresh thinking about how to stop viral outbreaks. One promising route has been suggested by Dr Anthony Fauci, who is one of the world's foremost authorities on infectious diseases and the longstanding director of the National Institute of Allergy and Infectious Diseases. In the face of the Zika virus epidemic a few years ago, Dr. Fauci remarked that "we need broad-spectrum antiviral drugs effective against whole classes of viruses" (widely quoted in media; see also ref. ${ }^{17}$ ). Unfortunately, now as we face the COVID-19 pandemic, we are still far away from this goal and more efforts need to be placed on advancing promising classes of broad-spectrum antiviral drugs as part of pandemic preparedness plans that fit within the VUCA concept to reduce risk and uncertainty ${ }^{18}$. Rather than investing time and resources into developing an antiviral drug to target a single virus, an effective broad-spectrum antiviral strategy would provide a multipurpose solution that could work against many viruses, including emerging ones ${ }^{19}$. Such developments would greatly improve pandemic preparedness by enabling potentially viable countermeasures even at relatively early stages of emerging outbreaks. One promising example is interferon-lambda (IFN- $\lambda$ ). IFN- $\lambda$ is a type-III interferon and is the body's first line defense against viruses ${ }^{20}$. Its receptors are largely limited to epithelial cells, including the lungs, liver and gastrointestinal tract, and it has broad spectrum in vitro and in vivo antiviral activity ${ }^{21}$. IFN- $\lambda$ has similar post-receptor antiviral signalling to type-I interferon-alpha (IFN- $\alpha$ ), but with less induction of inflammation, which may be particularly important in infections like COVID-19 where a major driver of the pathology is the cytokine storm ${ }^{22}$.

\section{Virus targeting strategies}

While each virus type has distinct structural features, many medically important viruses often share important qualities that can provide the basis for broad-spectrum antiviral targeting (Fig. 2). For example, many viruses have similar patterns of viral genome replication within infected cells, which has led to the development of the class of antiviral replication inhibitors ${ }^{23}$. Various nucleotide and nucleoside analogues have been developed to treat infections caused by many types of viruses ${ }^{24,25}$. This approach has proven clinically useful; however, viral genome replication is an inherently error-prone process and targeted viral proteins can quickly mutate to potentially render replication inhibitors obsolete if not used judiciously ${ }^{26}$. In terms of widely used countermeasures, it is thus unlikely that replication inhibitors alone will find sustained footing in the long term and they only work inside already infected cells. 

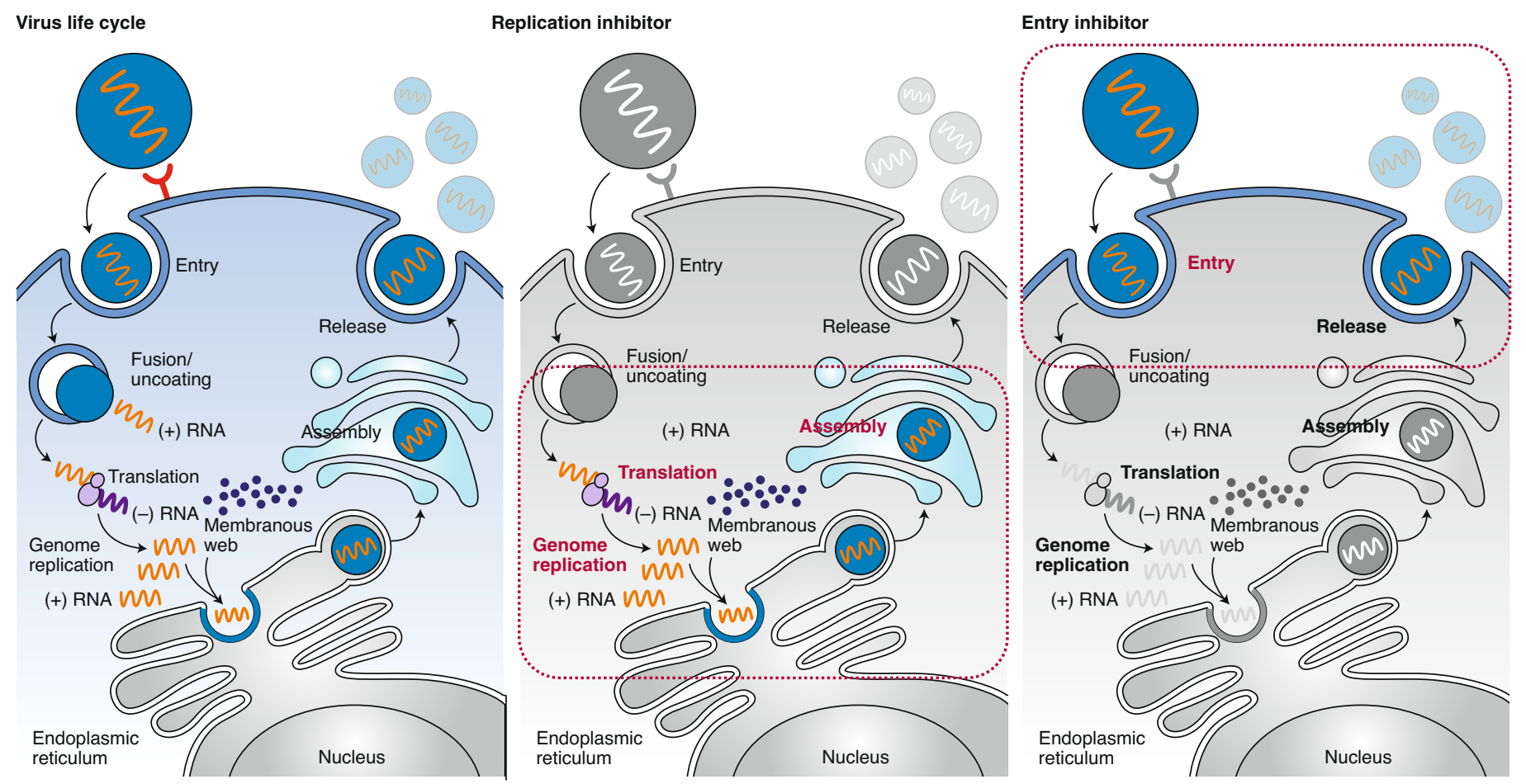

Fig. 2 | Classes of antiviral strategies. Viruses depend on infecting cells to support viral genome replication and the production of new virus particles. Thus, infection is a key part of the virus life cycle. Replication inhibitors are antiviral drugs aimed at stopping viral genome replication to curtail production of new virus particles. They work within already-infected cells. By contrast, entry inhibitors interact with existing virus particles outside of cells and prevent infection. They help to reduce viral loads and have proven useful in improving prophylactic and therapeutic outcomes.

\section{Direct targeting of virus particles}

Another emerging broad-spectrum antiviral strategy involves direct extracellular targeting of existing virus particles as opposed to stopping the production of new virus particles. This strategy can prevent infections and has demonstrated tremendous promise with the rise of antiviral antibodies that potently inhibit specific types of virus particles by binding to viral antigens on the virus particle surface $^{27}$. In some cases, antiviral antibodies can target highly conserved epitopes found across multiple viruses, and antibody-based treatments have emerged as one of the leading clinical options being explored for antiviral prophylaxis and therapy ${ }^{28}$. Materials science and engineering have also played important roles in devising new antiviral strategies to directly inhibit virus particles. Antiviral nanoparticles coated with flexible linkers mimicking heparan sulfate proteoglycans - a critical cell surface receptor used by viruses to bind to and infect cells in numerous cases have been shown to inhibit many viruses, such as herpes simplex, human papilloma, respiratory syncytial and dengue ${ }^{29}$.

Many viruses also contain a lipid bilayer coating, called an envelope, that surrounds virus particles, and its physicochemical disruption can abrogate viral infectivity ${ }^{30,31}$. This Achilles heel of enveloped viruses is especially noteworthy because a large fraction of recent viral outbreaks has been caused by enveloped viruses. Several types of small molecules ${ }^{32,33}$ and amphipathic peptides $^{34}$ have been reported to inhibit viral envelopes of various viruses such as human immunodeficiency virus, hepatitis $\mathrm{C}$ virus, Zika, dengue, yellow fever, chikungunya and Ebola. Since the lipid envelope is derived from host cell membranes and not the viral genome, it is anticipated that there is also a higher barrier to the emergence of drug-resistant virus strains $s^{35}$, which would increase the viability of broader antiviral prophylactic efforts. Importantly, coronaviruses, including the one causing COVID-19, are also enveloped viruses and thus potentially amenable to such approaches. However, it will require greater collaboration and crosstalk between materials scientists and clinician-scientists to realize the potential of such approaches and also to refine our mechanistic understanding of how these materials science-inspired antiviral strategies work in vivo. Engineering approaches can accelerate the broad-spectrum antiviral drug discovery and innovation stages but they need to be integrated together with preclinical drug development to enable lead candidates to reach clinical testing stages, as already seen with other classes of broad-spectrum antivirals.

\section{Stopping critical host cell functions} Another strategy designed to both decrease the potential for the emergence of resistance, as well as increase the potential for broad-spectrum activity, is to target the host cell functions on which viruses depend. This strategy has been driven by research in molecular virology and reached more advanced stages of the drug development progress so far, with compelling potential advantages over existing antiviral strategies. Thus, it provides a successful blueprint for broad-spectrum antiviral strategies developed from a materials science and engineering angle. In general, using small molecule inhibitors that target a host cell factor that is not under genetic control of the virus can present a more difficult evolutionary task for the virus to escape drug susceptibility. This approach contrasts with direct-acting antivirals that can bind a viral enzyme with high affinity, and where a single point mutation at the drug's binding 
site can result in loss of drug efficacy. For example, broad-spectrum kinase inhibitors, which have been approved for anticancer therapy, have demonstrated the potential to impair intracellular viral trafficking and thus inhibit a wide range of viruses, such as hepatitis $\mathrm{C}$, dengue and Ebola, that depend on this particular host cell function ${ }^{36,37}$. Human clinical trials have also shown that pharmacologic inhibition of a host target (for example, farnesyltransferase) can have a high barrier to resistance, with no patient at any point showing evidence of virus resistance even after months of treatment ${ }^{38,39}$. Another advantage of such a host cell targeting strategy is that many viruses may have evolved to depend on access to the same host cell function. For example, the host cell lipid kinase PI4KIII $\beta$, and its phosphorylated lipid products, are essential for the replication of many viruses (for example, hepatitis $\mathrm{C}$, rhinovirus and enterovirus $)^{40}$, and have also been implicated in host cell entry of SARS coronavirus (SARS-CoV).

\section{Learning from today for tomorrow}

Undoubtedly, we will face more viral outbreaks in the future and it is likely that at least some of them will be caused by viruses that are unknown, or at least understudied, today. Being prepared to confront these unknown unknowns with broad-spectrum antivirals either directly targeting virus particles, stopping critical host cell functions or enabling other mechanistic possibilities would significantly boost our current capabilities to ward off pandemics and dramatically reshape the landscape of antiviral drug development and what we view as 'druggable'. Most importantly, the above approaches can allow us to be proactive, instead of reactive, to emerging viral threats.

Within this scope, we believe that materials science can play an important role in enhancing our capabilities to stop future viral pandemics, including aiding the conceptual and practical development of broad-spectrum antiviral strategies. For example, the development of new antiviral technologies that are designed to interface with virus particles on similar geometrical scales, such as cell-decoy nanoparticles and membrane-curvature-sensitive peptides, offer great potential to prevent cellular infections. While most currently existing broad-spectrum antivirals inhibit viral genome replication or host cell functions after virus infection of host cells has occurred, direct targeting of virus particles offers the potential to stop viruses before infection of host cells occurs. Such capabilities also have the potential to reduce virus infectivity levels, which could accelerate recovery while reducing transmission risks. These capabilities are even more attractive if they involve antiviral mechanisms with a high barrier to resistance development, such as targeting viral membranes.

Looking forward, exploring innovative broad-spectrum antiviral strategies is just the first step in how materials science and engineering can contribute to global efforts to combat emerging viral pathogens and ward off future pandemics. We also envision that other fields of materials science will be vital to areas such as creating anti-infective surface coatings, rapidly manufacturing medical equipment and consumables, and lab-on-a-chip diagnostic sensors. Despite the current challenges of the COVID-19 pandemic, we believe that a bright future lies ahead where scientists can unite together across disciplines to tackle some of the biggest problems of our time, including viral pandemics.

Nam Joon Cho@1凶 and Jeffrey S. Glenn ${ }^{2,3}$
${ }^{1}$ School of Materials Science and Engineering,
Nanyang Technological University, Singapore,
Singapore. ${ }^{2}$ Department of Medicine, Division
of Gastroenterology and Hepatology, Stanford
University School of Medicine, Stanford, CA, USA.
${ }^{3}$ Department of Microbiology and Immunology,
Stanford University School of Medicine, Stanford,
CA, USA.
$\varpi_{e-m a i l: \text { njcho@ntu.edu.sg; jsglenn@stanford.edu }}$

Published online: 19 May 2020

https://doi.org/10.1038/s41563-020-0698-4

References

1. Kelly, H. et al. PLoS ONE 6, e21828 (2011).

2. Coltart, C. E., Lindsey, B., Ghinai, I., Johnson, A. M. \& Heymann, D. L. Philos. Trans. R. Soc. B 372, 20160297 (2017).

3. Ferguson, N. M. et al. Science 353, 353-354 (2016).

4. Spiteri, G. et al. Eurosurveillance 25, 2000178 (2020).

5. Hoehl, S. et al. N. Engl. J. Med. 382, 1278-1280 (2020).

6. Paules, C. I., Marston, H. D. \& Fauci, A. S. JAMA 323, 707-708 (2020).

7. Keener, A. Nat. Med. https://doi.org/10.1038/d41591-020-00002-4 (2020).

8. Tetro, J. A. Microbes Infect. 22, 72-73 (2020).

9. Mulangu, S. et al. N. Engl. J. Med. 381, 2293-2303 (2019).

10. Wang, M. et al. Cell Res. 30, 269-271 (2020).

11. Kujawski, S. A. et al. Preprint at https://doi. org/10.1101/2020.03.09.20032896 (2020).

12. Chen, Z. et al. Preprint at https://doi.

org/10.1101/2020.03.22.20040758 (2020).

13. Mahevas, M. et al. Preprint at https://doi. org/10.1101/2020.04.10.20060699 (2020).

14. Tang, X. et al. Natl Sci. Rev. https://doi.org/10.1093/nsr/nwaa036 (2020).

15. Bennett, N. \& Lemoine, G. J. Harv. Bus. Rev. https://hbr.org 2014/01/what-vuca-really-means-for-you (2014).

16. Nature 578, 191 (2020)

17. Fauci, A. S. \& Morens, D. M. N. Engl. J. Med. 374, 601-604 (2016).

18. Wei, X., Li, X. \& Cui, J. Natl Sci. Rev. 7, 239-242 (2020).

19. Bekerman, E. \& Einav, S. Science 348, 282-283 (2015).

20. Broggi, A., Granucci, F. \& Zanoni, I. J. Exp. Med. 217, e20190295 (2020).

21. Lazear, H. M., Nice, T. J. \& Diamond, M. S. Immunity 43, 15-28 (2015).

22. Crotta, S. et al. PLoS Pathog 9, e1003773 (2013).

23. De Clercq, E. Nat. Rev. Microbiol. 2, 704-720 (2004).

24. Furuta, Y. et al. Antiviral Res. 100, 446-454 (2013).

25. Warren, T. K. et al. Nature 508, 402-405 (2014).

26. De Clercq, E. Nat. Rev. Drug Discov. 6, 1001-1018 (2007).

27. Hangartner, L., Zinkernagel, R. M. \& Hengartner, H. Nat. Rev. Immunol. 6, 231-243 (2006).

28. Walker, L. M. \& Burton, D. R. Nat. Rev. Immunol. 18, 297-308 (2018)

29. Cagno, V. et al. Nat. Mater. 17, 195-203 (2018).

30. Vigant, F., Santos, N. C. \& Lee, B. Nat. Rev. Microbiol. 13, 426-437 (2015)

31. Jackman, J. A., Shi, P.-Y. \& Cho, N.-J. ACS Infect. Dis. 5, 4-8 (2018).

32. Vincent, M. R. S. et al. Proc. Natl Acad. Sci. USA 107, 17339-17344 (2010).

33. Wolf, M. C. et al. Proc. Natl Acad. Sci. USA 107, 3157-3162 (2010).

34. Jackman, J. A. et al. Nat. Mater. 17, 971-977 (2018). 35. Speerstra, S. et al. Antiviral Res. 149, 164-173 (2018). 36. Bekerman, E. et al. J. Clin. Invest. 127, 1338-1352 (2017). 37. Schor, S. \& Einav, S. DNA Cell Biol. 37, 63-69 (2018) 38. Koh, C. et al. Lancet Infect. Dis. 15, 1167-1174 (2015). 39. Yurdaydin, C. et al. Hepatology 67, 1224-1236 (2018). 40. Cho, N.-J. et al. Gastroenterology 148, 616-625 (2015). 